\title{
Highlights from the Annual Meeting of the American Society of Clinical Oncology 2014 in Chicago - Expert Opinions Revisited
}

\author{
Chair: Michael P. Lux ${ }^{a}$ \\ Participants: Peter Kern ${ }^{b} \quad$ Cornelia Liedtke $^{c} \quad$ Volkmar Müller $^{d} \quad$ Matthias Siebert $^{\mathrm{e}} \quad$ Marc Thill $^{\mathrm{f}}$ \\ a Universitäts-Brustzentrum Franken, Frauenklinik, Universitätsklinikum Erlangen, Germany \\ ${ }^{b}$ Universitätsklinikum Essen und Brustzentrum Düsseldorf im Luisenkrankenhaus; Lehrkrankenhaus der Ruhr-Universität Bochum \\ St. Elisabeth-Hospital, Bochum, Germany \\ ' Klinik für Frauenheilkunde und Geburtshilfe, Universitätsklinikum Schleswig-Holstein / Campus Lübeck, Germany \\ ${ }^{d}$ Klinik für Gynäkologie, Universitätsklinikum Hamburg-Eppendorf, Germany \\ e Universitäts-Frauenklinik, Operative Gynäkologie and Gynäkologische Onkologie, Universitätsspital Basel, Switzerland \\ ${ }^{f}$ Klinik für Gynäkologie und Geburtshilfe, Agaplesion Markus Krankenhaus, Frankfurt/M., Germany
}

The Annual Meeting of the American Society of Clinical Oncology (ASCO) 2014 May 30 to June 3 at McCormick Place in Chicago, Illinois, was again both the largest meeting in oncology, and also overwhelming in the variety of scientific presentations as well as of educational and other sources of information. Moreover, it was a special meeting - ASCO celebrates 50 years of advancing progress against cancer. The meeting was founded at a small lunch meeting on April 9, 1964. At the time of the first meeting, the landscape of cancer was ominous: cancer was widely regarded as a monolithic, largely untreatable disease. Much has changed since 1964. Today cancer is treated interdisciplinarily with modern therapeutic concepts. Efficacy is increasing and side effects become less and manageable. Especially gynecological cancers and breast cancer are in the focus of research and clinical trials. In Germany, these cancers affect 97,480 women every year, $43 \%$ of all cancer cases in women. Thus, gynecology has a special value in oncological care. Gynecological oncology and mastology have evolved dramatically in several directions corresponding to their importance in oncological care. This concerns modern diagnostics on which individual treatment plans are based (e.g. genetic subtypes in breast cancer), as well as the current multimodal and interdisciplinary treatment concepts. These aspects are also important for the ASCO - numerous results from clinical trials and further scientific data have been presented at this year's meeting.

We asked 5 German experts about their bottom line of the ASCO 2014 meeting on 5 main questions concerning breast cancer.

\section{KARGER}

Fax +497614520714

Information@Karger.com

www.karger.com (c) 2014 S. Karger GmbH, Freiburg

$1661-3791 / 14 / 0094-0287 \$ 39.50 / 0$

Accessible online at:

www.karger.com/brc

\section{Question 1: What Was Your Personal Highlight of the ASCO 2014 in Chicago?}

Liedtke: If you had asked me before this year's ASCO meeting had started, I probably would have mentioned the results of the ALTTO trial. However, since this study has to be called 'negative', I am afraid that regarding breast cancer treatment I did not see one single highlight but rather once again many small pieces of the final puzzle. These pieces, however, are almost as important as the results of large randomized trials since they may provide us with novel insights into the biology of breast carcinoma and thereby may provide the basis for novel therapeutic concepts.

Thill: In my opinion, it is not possible to define a single highlight and the so-called highlights presented were negative (ALTTO trial) or not really mature results (SOFT/TEXT trials). Data that I would highlight have been collected in premenopausal patients. The first trial was the pooled analysis of the SOFT and TEXT trials with an improvement in diseasefree survival (DFS) of premenopausal patients who were treated with exemestane and triptorelin compared to the combination with tamoxifen. These are surprising results as we already learned from the ABCSG-12 study that the combination of an aromatase inhibitor and a GnRH analogue has no benefit in premenopausal patients. However, a lot of data from the SOFT trial are lacking and the time of accrual was very long. Therefore, these are currently no practice changing data in my opinion. The second highlight was the POEMS 
study which has compared a cyclophosphamide containing chemotherapy with and without goserelin to prevent premature ovarian failure. The results presented by Halle Moore show, besides a surprisingly high pregnancy rate for the goserelin arm, an improved DFS and overall survival (OS) with goserelin. Due to these results, I think, it is an option to offer ovarian function suppression (OFS) for hormone-receptor negative patients who desire future fertility.

Müller: The oral presentation of the first results of BRCA mutation analysis from the triple negative (TNBC) cohort of the GeparSixto neoadjuvant trial is my personal highlight of the meeting. The presented analysis examined the correlation of germline BRCA alterations and family history for breast and ovarian cancer with the pathological complete response (pCR) rate in patients with TNBC. This question is of clinical relevance since there is a need for improvement of treatment outcome especially in TNBC but a lack of predictive factors for an increased benefit from carboplatin containing therapies. Alterations of BRCA or RAD50/51 were detected in at least $15 \%$ of the participants with TNBC in the GeparSixto trial. Patients with no further risk factors achieved a pCR rate of $40 \%$. The pCR rate tended to be higher in patients with family history of breast and ovarian cancer $(49 \%, p=0.21)$ or germline BRCA/RAD alterations $(55 \%, \mathrm{p}=0.11)$. The highest pCR rate was observed in patients with both (64\%). This indicates a special biology of the tumor of these patients and might help to optimize future treatment.

Siebert: One personal highlight of the ASCO 2014 in Chicago was the reflection on global oncological questions by the charismatic Nobel prize and this year's ASCO award winner Harald zur Hausen simply using bibliographical references such as 'Wikipedia'. Medicine does not necessarily involve complex cellular processes, but can sometimes be basic and simple. One main statement was that the consumption of red meat seems to be linked with colorectal cancer and other tumor entities due to virus interactions with carcinogens formed during cooking.

Kern: The Science of Oncology Award of the ASCO Annual Meeting 2014 granted to Harald zur Hausen for his lifetime achievements and outstanding lecture 'Do some Human Cancers Originate from Infections Transmitted from Domestic Animals?' was my personal highlight of this ASCO Annual Meeting. I had the opportunity to encounter his research activities on this field at ESGO in Belgrade in the year 2009, when he already suggested an association of gastric, pancreatic, colon, breast, and lung cancer citing numerous cohort and case control studies. Regarding premenopausal women with breast cancer, he presented 14 consistently positive studies -4 cohort trials and more than 7 case cohort trials - which showed an odds ratio for risk by consumption of red meat of 1.11 (case cohort) to 1.57 (case control). In his presen- tation he demonstrated own hypotheses by exploring the incidence and prevalence of cancer subtypes in various countries and their respective habits of eating red meat, followed by virological analyses and the identification of polyoma viruses associated with the carcinogenesis of the tumors mentioned above. Similarly, like the discovery of the association of the HPV high-risk infections and the carcinogenesis of cervical cancer was initially rejected by the scientific community, however later awarded with the Nobel prize, zur Hausen plans to work on a vaccination for meat cattle. Until a special vaccination is available, considerations have to be made how to deal with zur Hausen's findings of red meat consumption with regard to daily dietary recommendations.

\section{Question 2: Which Changes Do You See for the Neoadjuvant and Adjuvant Treatment of Breast Cancer Patients in the Coming Year That Are Triggered by the AsCO Meeting?}

Liedtke: Pagani and colleagues presented the results of the TEXT/SOFT joint analysis which demonstrated that premenopausal patients with breast cancer treated with aromatase inhibitors and OFS had a superior outcome (regarding disease-free, distant disease-free and breast cancer-free survival) compared to patients treated with tamoxifen and OFS. At first glance, these results appear practice changing. However, one has to acknowledge that there is a lack of comparisons with today's standard therapy, i.e. tamoxifen alone against these 2 OFS based combinations, before we can make a judgment regarding a potential novel standard therapeutic approach. However, in patients with contraindications against tamoxifen, TEXT/SOFT represent an additional piece of data that OFS in combination with an aromatase inhibitor may be a safe alternative for premenopausal breast cancer patients.

Müller: One surprising result was the presentation by Pagani et al. about the first results of the TEXT and SOFT trials. With this joint analysis of 4,690 premenopausal patients, the comparison of tamoxifen versus exemestane, each given in combination with OFS for 5 years was also published in the New England Journal of Medicine on the same day. With a median follow up of 5.7 years, a significant increase of the recurrence-free interval was observed with a hazard ratio of 0.66 and an absolute benefit of $4 \%$. Also surprisingly for me, the quality of life was similar in both treatment arms. My feeling is that patients experience more side effects with aromatase inhibitors and OFS compared to tamoxifen and OFS. Regarding the follow up, no difference in overall survival was observed. In my opinion, the combination of exemestane and OFS is not the new standard due to the lack of survival advantage and possible relevant side effects. The role of OFS in addition to tamoxifen compared to tamoxifen alone still remains unclear since this treatment arm was not presented. However, 
it is reassuring to know we can offer this treatment to patients that have a contraindication against tamoxifen.

Thill: This year's ASCO confirmed that bevacizumab does not achieve any survival benefit in the adjuvant setting. Kathy Miller presented the data of the E5103 trial. Neither DFS nor OS was improved by the addition of bevacizumab to an anthracycline/taxane containing regimen. Similar results have been presented by the BETH and BEATRICE trials. However, it is one of my standard compounds in the treatment of metastatic breast cancer (mBC). Regarding the neoadjuvant setting, Gunter von Minckwitz showed that the highest pCR rate amongst the triple negative group of the GeparSixto trial could be achieved in patients with both a family history of breast and ovarian cancer and alterations in BRCA/RAD. These data were confirmed by Paluch-Shimon and colleagues who described the highest $\mathrm{pCR}$ rate in BRCA1/2 patients, but no prediction of the relapse-free survival by $\mathrm{pCR}$.

It is getting more and more important to test TNBC patients for BRCA mutations. However, we also have to consider the currently known Lehmann subtypes of TNBC which predict the response to neoadjuvant chemotherapy. Certainly, the differentiation in Lehmann subtypes has to be done by our pathologists in the future.

Siebert: The addition of carboplatin to the cytostatic therapy in TNBC - especially in patients with BRCA-mutated or hereditary disease - as shown by Gunter von Minckwitz with the results of the GeparSixto trial - demonstrated an increase of more than $20 \%$ in pCR. In future, the 2014 ASCO Meeting will surely trigger this change in neoadjuvant (and maybe adjuvant) treatment of this group of breast cancer patients.

Kern: Regarding the neoadjuvant setting, I see changes in the treatment of TNBC patients with familial history of breast and/or ovarian cancer with and without germline mutations of BRCA/RAD towards the inclusion of carboplatin to an anthracycline and taxane containing regimen. A pCR rate of $66.7 \%$ is achieved even with a strict definition of pCR (ypTOypN0) and with regard to both conditions. Carboplatin achieves an increase of $23.2 \%$ of the pCR rate, and in case of a familial history without mutation an increase of $26.7 \%$ with a pCR rate of $57.5 \%$. Even without any of these conditions an increase of pCR rate of $11.5 \%$ (from 34.5 to $46.0 \%$ ) was detected; but without statistical significance, maybe due to the lower caseload. These findings correspond well with the overview of international data from the lecture of Melinda Telli (Stanford University), as well as with our own data which we presented at ASCO 2010 and published recently. Gunter von Minckwitz has proposed an effective carboplatin containing regimen with the GeparSixto trial, likewise William Sikov with the CALBG study 40603 at San Antonio Breast Cancer Symposium 2013, which may be chosen with regard to the respective tolerability of the patient.
In the adjuvant setting, I consider the data of Chiara O'Sullivan as confirmation not to withhold trastuzumab from patients with Her2/neu-positive breast cancers smaller than 2 $\mathrm{cm}$, since a benefit is conveyed in the hormone receptor-positive and -negative setting. She compared the trastuzumab containing arms with no trastuzumab in patients with tumors $\leq 2$ $\mathrm{cm}$ from 5 randomized trials (HERA, NCCTG N9831, NSABP B31, PACS 04, and FinHER). Patients with smaller tumors benefited substantially - both in terms of DFS and OS - from trastuzumab therapy. However, the authors indicated that almost all patients in these trials had T1c disease and positive lymph nodes, which represents a selected group of patients.

Eric P. Winer discussed his thoughts on Her2/neu-positive breast cancers $<1 \mathrm{~cm}$ and presented his 'Eric's Equation'. He suggests that pT1a tumors - if hormone receptor-positive should to be treated without trastuzumab and chemotherapy. I asked him whether he would also leave these tumors untreated in case of disseminated Her2/neu-positive tumor cells (DTC) of the bone marrow. He replied that he did not perform a bone marrow aspiration for DTC detection so far, but agreed that also the data from the German SUCCESS trial have demonstrated the negative prognostic value of positive DTCs which could contribute to a worse prognosis even in these smaller tumors.

\section{Question 3: In which Way Can the ASCO Meeting Influence the Future Treatment of Metastatic Breast Cancer?}

Müller: I did not realize practice changing results for the therapy of patients with metastatic breast cancer.

Liedtke: I have a feeling that every breast cancer meeting introduces one novel agent to the broader audience. This year's new drug was trebananib, an anti-angiogenic small molecule which has been analyzed in combination with trastuzumab and paclitaxel in the treatment in Her2/neu-positive metastatic breast cancer patients as part of a phase Ib clinical trial. This agent demonstrates promising response rates.

Thill: Many new compounds were presented, partially with promising but also with negative results. Combination therapies to overcome endocrine resistance will be very valuable in future. Promising approaches are the inhibition of CDK 4/6, FGFR or PI3K. No data were presented to change our current treatment of the triple negative subtype. However, the use of homologues recombinant deficiency (HRD) assays help to identify sporadic triple negative tumors which are similar to BRCA1/2 mutation associated cancer and react to platinum salts. Moreover, it is crucial to identify further biomarkers and driver mutations in the future. However, the SAFIR 01 trial presented current difficulties. 
Siebert: Recently we observed rapid progress especially in the management of advanced Her2/neu-positive breast cancer. Results from the ongoing MARIANNE trial suggest that trastuzumab emtasine (or 'T-DM1') has so far the potential to change the first-line therapy standard for Her2/neu-positive metastatic breast cancer patients due to supposed equivalent effectiveness combined with minimal toxicity.

Kern: In the Her2/neu-negative, hormone sensitive, metastatic setting, there is an evolving role of CDK 4/6 inhibition (palbociclib) which resulted in a 4 times prolonged progression-free survival in studies so far. In the Her2/neu-positive, metastatic setting, double targeting has also markedly prolonged progression free survival, and - by the optimal sequence of Her2/neu directed therapies - rendered breast cancer a chronic disease which is controllable often almost over a decade. As Shanu Modi from the Memorial Sloan Kettering Cancer Center has pointed out, multiple Her2/neu directed therapies are in development (neratinib, afatinib, HSP90 inhibitors, PI3K inhibitors, such as BKM 120 and BEZ235), which can potentially add further years to the survival of patients in the metastatic setting.

\section{Question 4: Which Results of Clinical Trials Presented at the ASCO Meeting Were Most Disappointing for You?}

Liedtke: I guess that most of the audience would agree that the presentation of the results of the ALTTO trial were the most disappointing result of this year's ASCO. Although the combination of lapatinib and trastuzumab has been shown to be more effective than either substance alone in the majority of studies in the metastatic and neoadjuvant setting, ALTTO demonstrated that the addition of lapatinib to trastuzumab did not enhance DFS in patients with Her2/neu-positive breast cancer in the adjuvant setting. Although there are several explanations for these results, such as dose reductions of lapatinib due to toxicity, trastuzumab in combination with chemotherapy remains standard of care unless the results of ongoing trials such as APHINITY using pertuzumab will be presented.

Müller: The results of the adjuvant ALTTO trial were disappointing in some ways. The addition of lapatinib to trastuzumab in the adjuvant treatment of Her2/neu-positive patients did not improve outcome. Importantly, the entire patient cohort had an excellent outcome with a 4-year DFS rate between 86 and $88 \%$. I think that the study does not allow the conclusion that the concept of dual Her2/neu blockade is not more effective than trastuzumab treatment alone. The combination of other Her2/neu targeting drugs and/or a better selection of high-risk patients could provide an improvement in outcome. This is currently addressed by several ongoing trials.
Thill: In my opinion, the most disappointing results were the long awaited results of the ALTTO trial. We already have excellent results for a dual Her2/neu blockade in the metastatic (CLEOPATRA) and in the neoadjuvant setting (NEOSPHERE). But, unfortunately, these excellent results could not be confirmed by the ALTTO study which has evaluated the use of trastuzumab and lapatinib. More than 8,000 patients were recruited. Both - trastuzumab in combination with lapatinib and trastuzumab followed by lapatinib - presented no significant difference in DFS. Besides the low-risk patient population, it was impressive to see an OS of $94-95 \%$ after 4.5 years due to the use of trastuzumab. However, the ALTTO results have to make us contemplative why results from the metastatic and the neoadjuvant setting are not reproducible in in the adjuvant situation.

Siebert: 'Disappointing' is a quite severe term in my opinion: The 'off' for the combined targeted therapy with trastuzumab and lapatinib analyzed by the ALTTO trial was not this unexpected since own experiences showed a high rate of toxicity at a questionable (now an objectively unproven) benefit. My personal surprise is that we now discuss a shorter and more expensive alternative endocrine therapy with even higher side effects in premenopausal patients with a hormone sensitive breast cancer as a result of the TEXT trial. These results are in contrast to our new guideline which recommends an extended therapy up to 10 years.

Kern: The ALTTO trial was most disappointing, as it demonstrated no role for double targeting with the addition of lapatinib to trastuzumab in the adjuvant setting. This also corresponds with the role of lapatinib in the Neo-ALTTO trial.

\section{Question 5: What Was the Most Important Contribution on Prognostic or Predictive Factors in Your Opinion?}

Liedtke: I was excited to see the analysis of the GeparSixto trial presented by Gunter von Minckwitz and colleagues regarding the association between the BRCA mutation status and the response to neoadjuvant platinum containing chemotherapy in patients with TNBC. Although the majority of attendants - including me - had a feeling that the benefit of platinum was confined to patients with hereditary carcinoma, we were surprised by the results demonstrating that BRCA/ RAD mutations alone did not predict a significantly better response to platinum-containing chemotherapy (pCR 43.5\% vs. $66.7 \%, p=0.13$ ). Interestingly, the authors observed an increased $\mathrm{pCR}$ rate by carboplatin among patients without mutation but positive family history (pCR $30.8 \%$ vs. $57.5 \%$, p $=0.02)$. These results, however, remain to be reproduced and, most of all, explained biologically. The AGO recommends using platinum containing chemotherapy among pa- 
tients with TNBC ('+'), however, there are no data yet as to whether there are certain subgroups that show a higher response.

Müller: Carsten Denkert presented results from the GeparSixto trial evaluating mRNA markers associated with the immune system function. This study is important in the context of a growing interest in the immune system for tumor response to chemotherapy. The authors and other groups have observed a prognostic and predictive impact of tumor infiltrating lymphocytes. In their work presented at ASCO, they evaluate different states of immune activation by mRNA markers. In conclusion, they found that the examined immune suppressing markers are expressed together with pro-immune markers, suggesting a feedback activation of immunosuppressive pathways and immune reaction. Almost all of the examined markers had a predictive role for therapy response, underlining an important role of the immune system.

Thill: Currently numerous different prognostic and predictive factors are under intense evaluation, but not really integrated in clinical trials. Therefore, one of the most important contributions regarding prognosis and prediction is the interim analysis of the ADAPT trial. Oleg Gluz et al. showed that the Ki67 value dropped down to $<10 \%$ in $70 \%$ of the hormone-receptor positive, Her2/neu-negative breast cancer patients with an Oncotype $\mathrm{DX}^{\circledR}$ recurrence score between 12 and 25. Potentially, this is the group of patients in which chemotherapy can be avoided in future. The most promising contribution was presented by Carsten Denkert who described a correlation between immune subtypes and the response to chemotherapy in terms of a link between immune markers and pCR. However, validation studies are needed.

Siebert: There were at least 2 important contributions about prognostic or predictive factors in my opinion: The PAM50 intrinsic subtyping showed a significant prognostic effect on relapse-free survival breast cancer patients treated with chemotherapy. Additionally, the predictive effect of tumor infiltrating lymphocytes (TILs) and lymphocyte predominant breast cancer was shown by an increased $\mathrm{pCR}$ rate in carboplatin treated TNBC (74\%) and Her2/neu-positive (78\%) breast cancer by Carsten Denkert as a result of the GeparSixto trial.
Kern: Harold Burstein presented the terminus of 'Precision Medicine' as 'the use of genomic, epigenomic, exposure and other data to define individual patterns of disease, potentially leading to better individual treatment' in the opening session of the ASCO Meeting 2014. The integration of genomic profiling to treatment decisions by first generation gene arrays, e.g. Oncotype DX, and genetic changes identified by next generation sequencing will overturn our so far existing treatment algorithms. Burstein showed that distinct subtypes of breast cancer have different repertoires of mutations, but there is no subtype specific highly mutated gene. So, parallel sequencing analyses of breast cancer tumors will be necessary to enable an individual portrait of each tumor before treatment.

\section{Participants}

Dr. Peter Kern

Universitätsklinikum Essen und

Brustzentrum Düsseldorf im Luisenkrankenhaus

Hufelandstraße 55

45147 Essen, Germany

Peter.Kern@uk-essen.de

PD Dr. med. Cornelia Liedtke

Klinik für Frauenheilkunde und Geburtshilfe

Universitätsklinikum Schleswig-Holstein / Campus Lübeck

Ratzeburger Allee 160

23538 Lübeck, Germany

Cornelia.Liedtke@uksh.de

Prof. Dr. med. Volkmar Müller

Klinik für Gynäkologie

Universitätsklinikum Hamburg-Eppendorf

Martinistrasse 52

20246 Hamburg, Germany

v.mueller@uke.de

Dr. med. Matthias Siebert

Universitäts-Frauenklinik

Operative Gynäkologie and Gynäkologische Onkologie

Universitätsspital Basel

Spitalstrasse 21

4031 Basel, Switzerland

matthias.siebert@usb.ch

PD Dr. med. Marc Thill

Klinik für Gynäkologie und Geburtshilfe

Agaplesion Markus Krankenhaus

Wilhelm-Epstein-Straße 4

60431 Frankfurt/M., Germany

marc.thill@fdk.info 